\title{
Variabilidad morfológica de variedades nativas de mora (Rubus sp.) en los Andes de Colombia
}

\author{
Morphological variability of wild blackberry (Rubus sp.) \\ cultivars in the Andes of Colombia
}

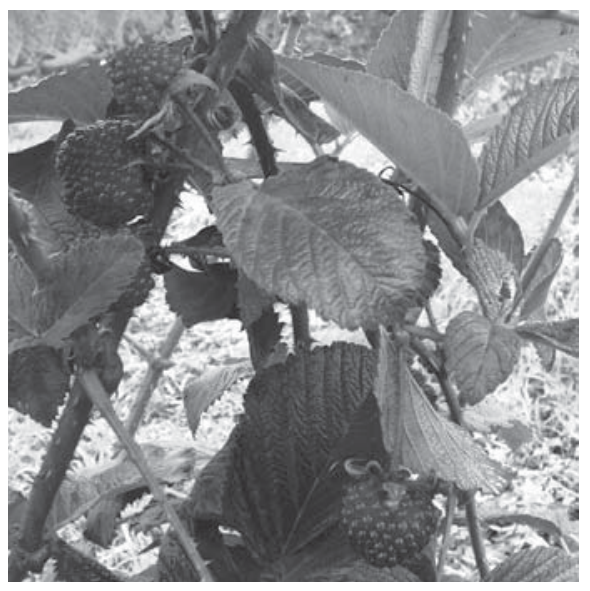

NATALIA ESPINOSA B..$^{1,5}$

GUSTAVO ADOLFO LIGARRETO M. ${ }^{2}$

LUZ STELLA BARRERO M. ${ }^{3}$

CLARA INÉS MEDINA C. ${ }^{4}$

Rubus macrocarpus Benth. en zona altoandina colombiana.

Foto: N. Espinosa B.

\section{RESUMEN}

Se estudió la colección del género Rubus compuesta por 31 entradas de accesiones nativas y cultivadas, existentes en el Banco de Germoplasma de Colombia, en su mayoría procedentes de departamentos productores de mora con el fin de estimar su variabilidad, mediante 38 descriptores entre cuantitativos y cualitativos en la descripción morfológica. Se encontró que las agrupaciones de accesiones coinciden con la determinación taxonómica convencional para especies del género Rubus; se confirma la accesión RG37 como un material intermedio a nivel morfológico entre la especie $R$. glaucus y las especies silvestres. Se estableció variabilidad fenotípica en los materiales Rubus y se identificaron agrupaciones dentro de $R$. glaucus que muestran algunas accesiones con mayor introgresión de características vegetativas de las accesiones silvestres y otras con características más adecuadas a la producción comercial, así como alguna interacción del departamento de origen en el comportamiento fenotípico de las entradas de $R$. glaucus.

Palabras clave adicionales: recursos genéticos, descriptores fenotípicos, diversidad genética, fenotipo.

1 Programa de Mejoramiento Genético en Arroz, Federación Nacional de Arroceros de Colombia (Fedearroz), Cali (Colombia).

2 Facultad de Ciencias Agrarias, Departamento de Agronomía, Universidad Nacional de Colombia, Bogotá (Colombia).

3 Centro de Investigación Tibaitatá, Corporación Colombiana de Investigación Agropecuaria (Corpoica), Mosquera (Colombia).

4 Centro de Investigación La Selva, Corporación Colombiana de Investigación Agropecuaria (Corpoica), Rionegro, Antioquia (Colombia).

5 Autor para correspondencia. nataliaespinosa@fedearroz.com.co 


\section{ABSTRACT}

The present work studied the genus Rubus collection of Colombia; it consists of 31 entries of native and cultivated accessions mostly from different blackberry producer regions. In order to estimate genetic variability 38 quantitative and qualitative descriptors were used in morphologic description. At the morphological level, it was found that groups of accessions match with the conventional taxonomic determination species from the genus Rubus. RG37 accession was established as an intermediate material at the morphological level between species $R$. glaucus and some wild Rubus material. Morphological characterization also recognized phenotypic variability in Rubus materials and identified clusters within $R$. glaucus showing some accessions with higher closeness of vegetative characteristics from wild accessions. Additionally, it identified others which are better suited in commercial production, as well as some interactions of the region of origin in the phenotypic behavior of $R$. glaucus entries.

Additional key words: genetic resources, phenotypic descriptors, genetic diversity, phenotype.

Fecha de recepción: 15-06-2016 Aprobado para publicación: 24-11-2016
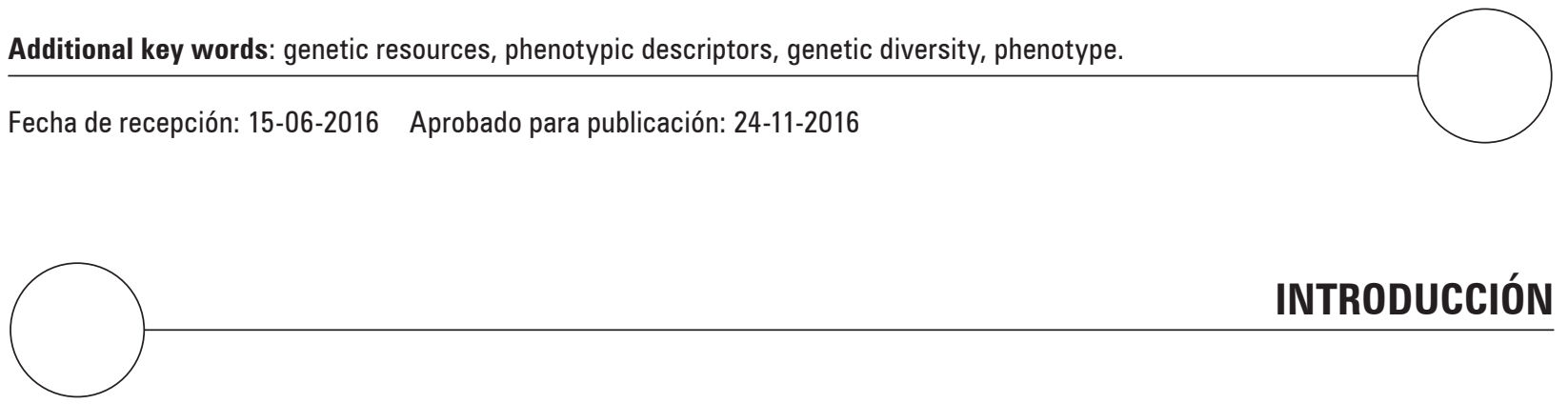

La mora pertenece al género Rubus, uno de los géneros de plantas más numerosos con aproximadamente 750 especies y con mayor adaptación ambiental ya que sus especies se encuentran distribuidas en todo el mundo, excepto en la Antártica (Alice y Campbell, 1999). La importancia del género se debe a varias especies frutícolas y ornamentales de valor económico y al interés ecológico por algunas arvenses (Alice y Campbell, 1999).

Las especies del género Rubus han acompañado la dieta humana desde la antigüedad en culturas de ambos hemisferios (Clark et al., 2007). Desde el siglo IV a.e.c. en Roma se consumían frutos frescos y bebidas de frambuesas y zarzamoras y sus hojas se utilizaban en la preparación de té con fines terapéuticos (Patel et al., 2004). De igual manera, los primeros reportes de los colonizadores españoles alrededor de los años 1500, indican la presencia de moras tanto en zonas insulares como en tierra firme del continente americano, las cuales describieron como plantas más vigorosas y en algunos casos con frutos de mayor tamaño que las especies europeas (Patiño, 2000). En los últimos años el interés en las moras y otras especies del género $R u$ bus ha crecido gracias a sus altos contenidos de antocianinas, compuestos fenólicos y flavonoides por lo que son reconocidas en medicina y terapeutica como retardantes de la oxidación de los tejidos causantes del envejecimiento físico y mental (Patel et al., 2004; Finn, 2008; Trivedi et al., 2016).
Los mayores esfuerzos en mejoramiento de especies Rubus se han hecho en las zonas templadas, a cargo de la Universidad de Arkansas (Clark et al., 2007), con el objetivo de desarrollar genotipos sin espinas, ramas erectas, frutos firmes y de mayor tamaño, alto rendimiento y en los últimos años en pro de buscar la fructificación de la rama primaria o principal, debido a que no produce frutos en el primer año de cultivo.

La Universidad de Arkansas evaluó la relación genética entre 32 cultivares de zarzamora norteamericanos, mediante análisis de pedigree, dilucidando hasta sus clones originarios. Se encontró que 19 clones contribuyen en la formación de los 32 cultivares; en particular, tres clones aportaron casi el $50 \%$ de los 32 cultivares, lo que evidencia una estrecha base genética (Stafne y Clark, 2004).

En los Andes de Colombia la mora pasó de ser una especie silvestre de consumo doméstico, a ser un cultivar de importancia comercial ganando rentabilidad e iniciándose su sistema de manejo agronómico. En consecuencia, la producción de mora en el país creció de $20.000 \mathrm{t}$ año-1 en 1992 a $90.000 \mathrm{t}$ año-1 en el 2008. En este mismo periodo, aumentó el área sembrada de 3.000 ha a 10.500 ha y a 11.673 en 2011, con rendimiento promedio alrededor de $8 \mathrm{t} \mathrm{ha} \mathrm{a}^{-1}$. En los departamentos con mayor producción Cundinamarca, Santander y Antioquia, en los últimos 5 años se han alcanzado rendimientos de hasta $15 \mathrm{t}$ $\mathrm{ha}^{-1}$ (MADR, 2012). 
El sistema de Bancos de Germoplasma de Colombia a cargo de la Corporación Colombiana de Investigación Agropecuaria (Corpoica), ha consolidado una colección ex situ de especies Rubus constituida principalmente por material colectado en campos de agricultores y algunas especies silvestres; sin embargo, hay poco conocimiento acerca de la diversidad genética presente en las especies Rubus colombianas. Si bien es cierto que se han adelantado algunos trabajos en algunas regiones del país con materiales Rubus tanto silvestres como cultivados (Marulanda y Márquez, 2001; Morillo et al., 2005; Zamorano et al., 2007; Marulanda et al., 2007), se hace necesario ampliar el conocimiento de colecciones de otras regiones de las cuales se carece de información y clarificar las estrategias de conservación, las necesidades adicionales de colecta y el uso del germoplasma en programas de selección y mejoramiento genético.

Con la finalidad de conocer y promover el uso del banco de germoplasma de especies Rubus colombianas, el objeto del presente trabajo fue describir la variabilidad genética presente en la colección de especies Rubus conservadas por Corpoica, mediante caracteres morfológicos para conocer su diversidad fenotípica.

\section{MATERIALES Y MÉTODOS}

En este estudio se utilizaron 31 accesiones de la colección de germoplasma ex situ de Rubus mantenidas en Corpoica, Centro de Investigación "La Selva" en Rionegro (Antioquia). La colección estuvo conformada por tres especies a saber: 29 accesiones $R$. glaucus, una accesión Rubus urticifolius y una de Rubus floribundus (Tab. 1). La determinación taxonómica de las accesiones de la colección de especies Rubus fue hecha con base en la clave de especies Rubus compendiada en la serie Flora del Ecuador (Romoleroux, 1996) y por expertos en el género Rubus, del Instituto de Ciencias Naturales de la Universidad Nacional de Colombia, sede Bogotá. Para identificar (ID) los materiales se utilizó una nomenclatura combinada de letras y números donde las letras indican la especie a la cual pertenece cada accesión así RG: $R$. glaucus, RF: $R$. floribundus y RU: $R$. urticifolius (Tab. 1).

En cinco plantas por accesión y con edad de tres años, se midieron 22 variables cuantitativas en estructuras reproductivas, de las cuales nueve, también se midieron en estructuras vegetativas por lo que suman 31 descriptores y 16 variables cualitativas adaptadas con base en los descriptores usados para caracterizar materiales Rubus del suroccidente colombiano (Zamorano et al., 2007) (Tab. 2).

Para el análisis estadístico de los datos cuantitativos se realizaron análisis de componentes principales y análisis de conglomerados con el programa SPAD Versión 5.5 (Decisia, 2003). Se seleccionaron los componentes con valor propio $\geq 1$ (Franco e Hidalgo, 2003). El programa SPAD utiliza el método de agrupamiento jerárquico de Ward para construir los dendrogramas, el cual busca maximizar la variación entre los grupos y minimizarla dentro de ellos (Ward, 1963). En el caso de los datos cualitativos se utilizó el programa MVSP 3.13 (Multivariate Statistical Package) (Kovach, 1999), para realizar el análisis de coordenadas principales ( $\mathrm{PCO}$, por su sigla en inglés) con base en el coeficiente de similitud de Gower (Gower, 1971). La conformación de los agrupamientos se realizó con el algoritmo de agrupamiento jerárquico UPGMA (Unweighted pair-group method arithmetic average).

\section{RESULTADOS}

\section{Análisis de descriptores cuantitativos}

El análisis de componentes principales de las variables de tipo reproductivo muestra que los primeros ocho componentes tienen valores propios superiores a $1 \mathrm{y}$ son los de mayor relevancia en la medida que agrupan alrededor de un $84,78 \%$ de la variación total de los datos originales. El primer componente principal constituye el 31,42\% de la varianza total explicada; de acuerdo con los datos de correlación las variables que más contribuyeron de manera positiva con este componente fueron longitud de peciolo en rama vegetativa y reproductiva, longitud del peciolulo en rama vegetativa y longitud del foliolo en rama reproductiva, otras variables que contribuyeron en menor proporción fueron la longitud del peciolulo en rama reproductiva, longitud del foliolo en rama vegetativa y el tamaño de las estípulas (Tab. 2). Por su parte, las variables número de aguijones en tallo reproductivo $y$ vegetativo fueron las que más contribuyeron a este componente de forma negativa. Lo anterior indica que el primer componente agrupa accesiones con peciolos, peciolulos, foliolos y estípulas largos y menor número de aguijones en tallos (Tab. 2).

Se observa en la tabla 2 que la distinción que se realiza entre ramas vegetativas y reproductivas, para las variables de mayor correlación con los cuatro primeros 
Tabla 1. Accesiones del banco de germoplasma de especies de Rubus spp. estudiadas.

\begin{tabular}{|c|c|c|c|c|c|c|c|}
\hline $\mathrm{N}^{0}$ & ID & No. Accesión Corpoica & Especie & Departamento & Municipio & Altitud (m) & Estado biológico \\
\hline 1 & RF36 & ILS 1817 & R. floribundus & Antioquia & Santa Rosa & 2.544 & Silvestre \\
\hline 2 & RG01 & ILS 2282 & R. glaucus & Caldas & Riosucio & 2.200 & Nativo \\
\hline 3 & RG02 & ILS 2288 & R. glaucus & Caldas & Villamaría & 2.200 & Nativo \\
\hline 4 & RG03 & ILS 2279 & R. glaucus & Caldas & Pácora & 2.000 & Nativo \\
\hline 5 & RG04 & ILS 2276 & R. glaucus & Caldas & Manzanares & 2.100 & Mejorado \\
\hline 6 & RG05 & ILS 2272 & R. glaucus & Antioquia & Guarne & 2.100 & Nativo \\
\hline 7 & RG09 & & R. glaucus & Desconocido & & & \\
\hline 8 & RG10 & ILS 2287 & R. glaucus & Caldas & Salamina & 1.900 & Nativo \\
\hline 9 & RG11 & ILS 2273 & R. glaucus & Caldas & Guática & 1.800 & Nativo \\
\hline 10 & RG12 & ILS 2370 & R. glaucus & Huila & & & \\
\hline 11 & RG13 & ILS 2269 & R. glaucus & Quindío & Córdoba & 1.900 & Mejorado \\
\hline 12 & RG14 & ILS 2283 & R. glaucus & Caldas & Salamina & 2.400 & Silvestre \\
\hline 13 & RG19 & ILS 2274 & R. glaucus & Antioquia & La Ceja & 2.300 & Nativo \\
\hline 14 & RG20 & ILS 2270 & R. glaucus & Antioquia & Don Matías & 2.430 & Nativo \\
\hline 15 & RG21 & ILS 2284 & R. glaucus & Caldas & Salamina & 2.100 & Nativo \\
\hline 16 & RG22 & ILS 2281 & R. glaucus & Antioquia & Envigado & 2.510 & Nativo \\
\hline 17 & RG23 & ILS 2286 & R. glaucus & Caldas & Salamina & 2.100 & Mejorado \\
\hline 18 & RG24 & ILS 2280 & R. glaucus & Caldas & Manizales & 2.430 & Silvestre \\
\hline 19 & RG25 & ILS 2285 & R. glaucus & Caldas & Salamina & 2.100 & Nativo \\
\hline 20 & RG27 & ILS 2268 & R. glaucus & Caldas & Manizales & 2.100 & Silvestre \\
\hline 21 & RG28 & ILS 2271 & R. glaucus & Antioquia & El Retiro & 2.500 & Nativo \\
\hline 22 & RG31 & ILS 1863 & R. glaucus & Nariño & La Cocha & 2.720 & Material de agricultor \\
\hline 23 & RG32 & ILS 2277 & R. glaucus & Valle & Tuluá & & \\
\hline 24 & RG38 & ILS 2324 & R. glaucus & Risaralda & Guática & 2.200 & Material de agricultor \\
\hline 25 & RG40 & ILS 2278 & R. glaucus & Antioquia & Jardín & 2.715 & Silvestre \\
\hline 26 & RG41 & ILS 1884 & R. glaucus & Antioquia & Medellín & 2.518 & Nativo \\
\hline 27 & RG43 & ILS 1857 & R. glaucus & Nariño & La Cocha & 2.810 & Material de agricultor \\
\hline 28 & RG45 & ILS 1861 & R. glaucus & Nariño & La Cocha & 2.720 & Material de agricultor \\
\hline 29 & RG47 & ILS 2362 & R. glaucus & Caldas & Manizales & & Nativo \\
\hline 30 & RG48 & ILS 2364 & R. glaucus & Santander & Pie de Cuesta & 2.150 & Nativo \\
\hline 31 & RU35 & ILS 1865 & R. urticifolus & Nariño & Sandoná & 1.839 & Silvestre \\
\hline
\end{tabular}

componentes principales no implica mayor relevancia en la diferenciación de accesiones Rubus spp.; ya que las variables tomadas en los dos tipos de ramas muestran correlaciones cercanas, como en el caso de longitud de peciolo donde la correlación es alta tanto en el caso de rama vegetativa como reproductiva.

En la figura 1 se observan las agrupaciones que se obtuvieron a partir del análisis de los 31 componentes principales utilizando todas las variables morfológicas en estudio, en el cual se conformaron tres grupos de cultivares. En el grupo uno se agruparon las dos especies silvestres evaluadas (R. urticifolius y $R$. floribundus), lo cual muestra sus diferencias morfológicas con respecto a la especie $R$. glaucus, evidenciadas en peciolos cortos, peciolulos y foliolos intermedios, un mayor número de aguijones en la hoja (promedio 26,58), mayor proporción de frutos cónicos y tallos de entrenudos largos (Tab. 3).

Con respecto a las agrupaciones 2 y 3 conformadas por accesiones de $R$. glaucus, el 2 se compone de accesiones con características más cercanas al grupo 1 de accesiones silvestres. En cambio, el grupo 3 conforma el brazo más distante en el análisis de agrupamientos. Por su parte, los cultivares de la especie $R$. glaucus se dividieron en los grupos 2 y 3 , los cuales tuvieron en común una menor longitud de peciolo, menor número de aguijones en hoja, entre 13 y 14; tallos con entrenudos cortos y menos frutos cónicos que las especies silvestres. El grupo 2 reunió seis accesiones de Antioquia y cinco de Caldas, de las catorce que lo 
Tabla 2. Correlaciones entre los descriptores de Rubus spp. y los cuatro primeros componentes principales.

\begin{tabular}{|c|c|c|c|c|}
\hline Descriptor & Componente 1 & Componente 2 & Componente 3 & Componente 4 \\
\hline \multicolumn{5}{|c|}{ Descriptores en estructuras vegetativas } \\
\hline Diámetro del tallo en ramas vegetativas & 0,27 & $-0,40$ & $-0,24$ & 0,22 \\
\hline No. de aguijones en un tallo vegetativo & $-0,58$ & $-0,10$ & $-0,49$ & $-0,29$ \\
\hline Longitud de entrenudos en un tallo vegetativo & $-0,18$ & 0,46 & $-0,58$ & 0,45 \\
\hline Tamaño de estípulas en un tallo vegetativo & 0,66 & 0,55 & 0,15 & 0,23 \\
\hline Longitud del foliolo terminal en un tallo vegetativo & $0,74^{*}$ & 0,44 & 0,15 & 0,26 \\
\hline Ancho del foliolo terminal en un tallo vegetativo & 0,69 & 0,48 & 0,15 & 0,13 \\
\hline Longitud del peciolo en un tallo vegetativo & $0,90^{*}$ & $-0,01$ & $-0,08$ & 0,21 \\
\hline Longitud del peciolulo en un tallo vegetativo & $0,82^{*}$ & 0,36 & 0,07 & 0,14 \\
\hline No. de aguijones en la hoja de un tallo vegetativo & $-0,06$ & $0,85^{*}$ & $-0,21$ & 0,01 \\
\hline \multicolumn{5}{|c|}{ Descriptores en estructuras reproductivas } \\
\hline Diámetro del tallo en ramas reproductivas DIAMTR & 0,17 & $-0,28$ & $-0,62$ & $-0,18$ \\
\hline Número de aguijones en un tallo reproductivo NAGTR & $-0,67$ & 0,16 & $-0,38$ & 0,00 \\
\hline Longitud de entrenudos en un tallo reproductivo LENR & 0,12 & 0,46 & $-0,45$ & $0,57^{*}$ \\
\hline Tamaño de estípulas en un tallo reproductivo TAMESTR & $0,73^{*}$ & 0,41 & 0,11 & $-0,19$ \\
\hline Longitud del foliolo terminal en un tallo reproductivo LFOLR & $0,81^{*}$ & 0,28 & 0,14 & $-0,20$ \\
\hline Ancho del foliolo terminal en un tallo vegetativo ANFOLR & 0,65 & 0,36 & 0,09 & $-0,41$ \\
\hline Longitud del peciolo en un tallo reproductivo LPECR & $0,92^{*}$ & $-0,02$ & $-0,08$ & $-0,05$ \\
\hline Longitud del peciolulo en un tallo reproductivo LPLULOR & $0,77^{*}$ & 0,40 & 0,09 & $-0,18$ \\
\hline Número de aguijones en la hoja de un tallo reproductivo NAGHR & 0,01 & $0,86^{*}$ & $-0,19$ & $-0,25$ \\
\hline No. de piezas de la corola NPCOR & $-0,09$ & 0,05 & 0,23 & 0,16 \\
\hline No. de piezas del cáliz NPCA & $-0,47$ & 0,52 & 0,22 & 0,25 \\
\hline Longitud del pétalo LPET & 0,05 & $-0,22$ & 0,50 & $-0,16$ \\
\hline Ancho del pétalo APET & $-0,43$ & 0,37 & 0,14 & $-0,40$ \\
\hline No. de frutos de forma primaria NFP & 0,07 & 0,24 & $-0,76^{*}$ & $-0,28$ \\
\hline No. de frutos de forma secundaria NFS & 0,24 & 0,41 & $-0,51$ & $-0,39$ \\
\hline Peso del fruto PFRUT & 0,63 & $-0,59$ & $-0,06$ & 0,04 \\
\hline Diámetro del fruto DFRUT & 0,61 & $-0,71$ & $-0,04$ & $-0,04$ \\
\hline Longitud del fruto LFRUT & 0,71 & $-0,53$ & $-0,22$ & 0,06 \\
\hline Diámetro del receptáculo DREC & 0,35 & 0,00 & $-0,30$ & 0,08 \\
\hline Longitud del receptáculo LREC & 0,66 & $-0,50$ & $-0,30$ & 0,09 \\
\hline No. de drupeolas NDRUP & 0,57 & $-0,50$ & $-0,30$ & $-0,08$ \\
\hline Rendimiento REND & 0,45 & 0,03 & 0,12 & $-0,40$ \\
\hline
\end{tabular}

*Descriptores con las correlaciones más altas.

Tabla 3. Valores promedio de las características discriminantes en las agrupaciones obtenidas por análisis de conglomerados de caracteres cuantitativos en Rubus spp.

\begin{tabular}{|l|c|c|c|}
\hline \multicolumn{1}{|c|}{$\begin{array}{c}\text { Descriptores cuantitativos que más aportan a la } \\
\text { variabilidad morfológica }\end{array}$} & $\begin{array}{c}\text { Grupo 1 } \\
\text { Especies silvestres }\end{array}$ & $\begin{array}{c}\text { Grupo 2 } \\
\text { R. glaucus cultivadas }\end{array}$ & $\begin{array}{c}\text { Grupo 3 } \\
\text { R. glaucus cultivadas }\end{array}$ \\
\hline Longitud peciolo en rama vegetativa (cm) & 7,38 & 10,08 & 11,19 \\
\hline Longitud peciolo en rama reproductiva(cm) & 8,11 & 8,89 & 12,90 \\
\hline Longitud peciolulo en rama vegetativa (cm) & 2,69 & 2,41 & 3,11 \\
\hline Longitud foliolo en rama reproductiva (cm) & 9,81 & 9,46 & 11,17 \\
\hline No. de aguijones hoja en rama vegetativa & 27,91 & 13,40 & 14,19 \\
\hline No. de aguijones hoja en rama reproductiva & 26,58 & 13,13 & 14,68 \\
\hline Tamaño de estípulas en rama reproductiva (mm) & 0,97 & 0,78 & 1,00 \\
\hline Número de frutos cónicos & 13,33 & 11,57 & 11,96 \\
\hline Longitud de entrenudos en rama reproductiva (cm) & 8,38 & 6,46 & 6,90 \\
\hline Número de piezas de la corola & 5,15 & 5,18 & 5,02 \\
\hline
\end{tabular}




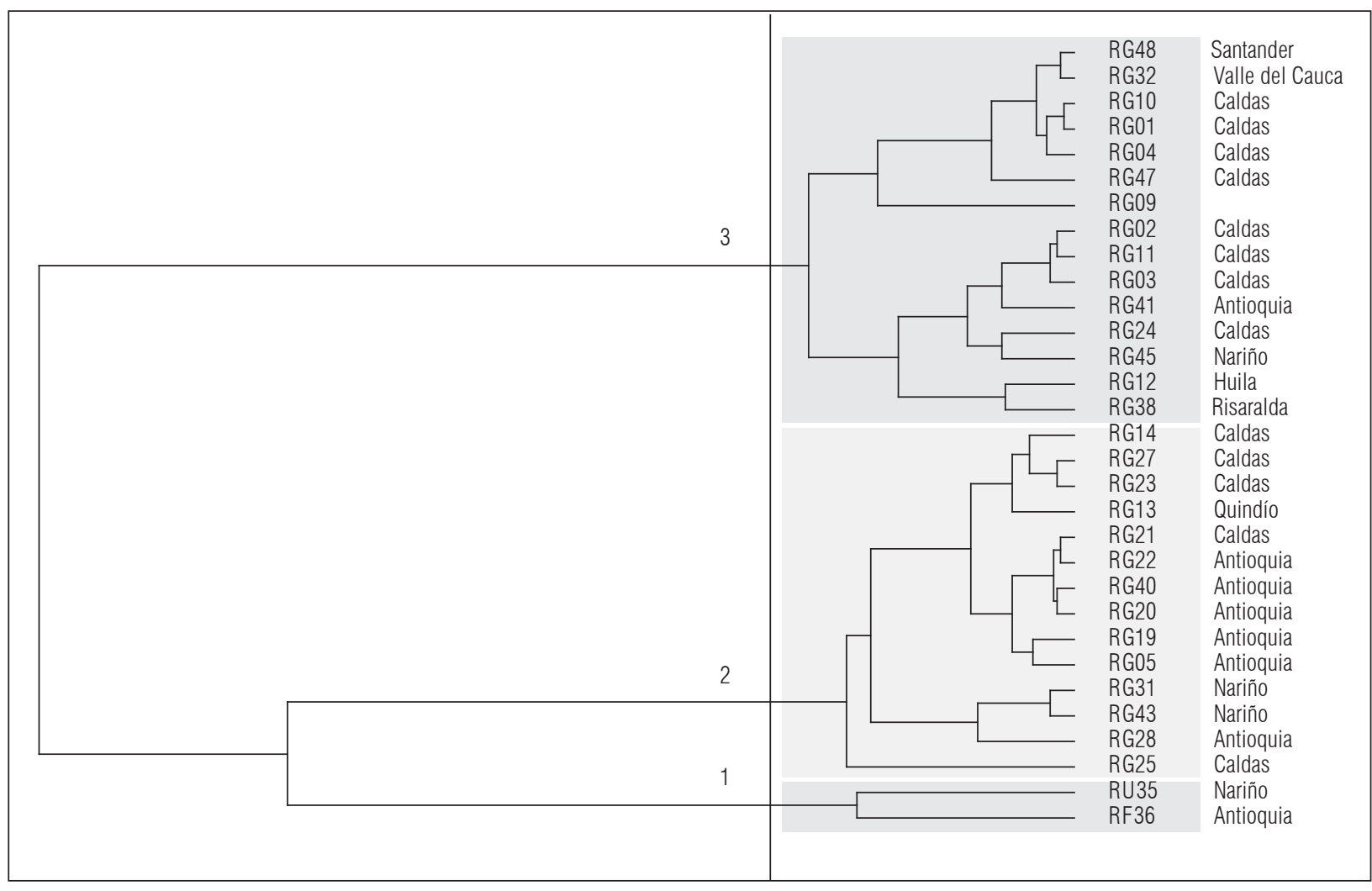

Figura 1. Dendrograma obtenido por análisis de componentes principales para 31 accesiones de Rubus spp. a partir de 31 variables cuantitativas. Análisis con SPAD 5.5.

cu: Cundinamarca; ri: Risaralda; sa: Santander; ca: Caldas; an: Antioquia; qu: Quindío; hu: Huila; na: Nariño; vc: Valle del Cauca.

componen; mientras que en el grupo 3 predominaron las accesiones colectadas en Caldas (Fig. 1; Tab. 3). En el grupo 2 clasificaron accesiones con peciolos $(8,89$ a $10,08 \mathrm{~cm})$, peciolulos $(2,41 \mathrm{~cm})$ y foliolos $(9,46 \mathrm{~cm}) \mathrm{de}$ longitud entre corta e intermedia, mientras que en el grupo 3 se asociaron materiales con peciolos $(11,19$ a $12,90 \mathrm{~cm})$, peciolulos $(3,11 \mathrm{~cm})$ y foliolos $(11,17 \mathrm{~cm})$ más largos. La accesión RG38, que se distingue por la ausencia de aguijones se asoció en el grupo 3 .

\section{Análisis de descriptores cualitativos}

En el trabajo no se consideraron los descriptores cualitativos correspondientes al estado reproductivo debido a que no pudieron ser tomados en todas las accesiones consideradas, porque no presentaron floración o fructificación durante el momento de evaluación o como en el caso de la accesión RG37, la cual presentaba floración pero no cuajado de fruto bajo las condiciones del C.I. La Selva en Rionegro, Antioquia. También se señala que en las variables cualitativas la distinción entre tallo reproductivo y vegetativo es insignificante en la diferenciación de los materiales de las especies Rubus spp. de la colección en estudio, debido a que no indican diferencias entre los dos tipos de tallo.

Con una similitud de 0,6 encuentran concordancia las especies silvestres $R$. urticifolius y $R$. floribundus con el conjunto de accesiones de materiales cultivados $R$. glaucus debido a que comparten la característica forma de la hoja ovada-elíptica. Con una similitud de alrededor de 0,89 se establecen cuatro agrupaciones de las accesiones Rubus (Fig. 2; Tab. 4).

Las dos accesiones correspondientes a especies silvestres ( $R$. urticifolius y $R$. floribundus) conforman el grupo 4 con una similitud de 0,77 entre ellas. Las características que las diferencian de la especie $R$. glaucus son la ausencia de cera en los tallos, los tallos acanalados, la presencia de pubescencia en los tallos, los aguijones tanto rectos como recurvados en tallo y hoja, las hojas son palmeadas o combinan palmeadas y trifoliadas, el margen de la hoja es serrado, la base de la hoja es obtusa, el ápice de la hoja es agudo y el color del envés es verde (Fig. 2; Tab. 4). 


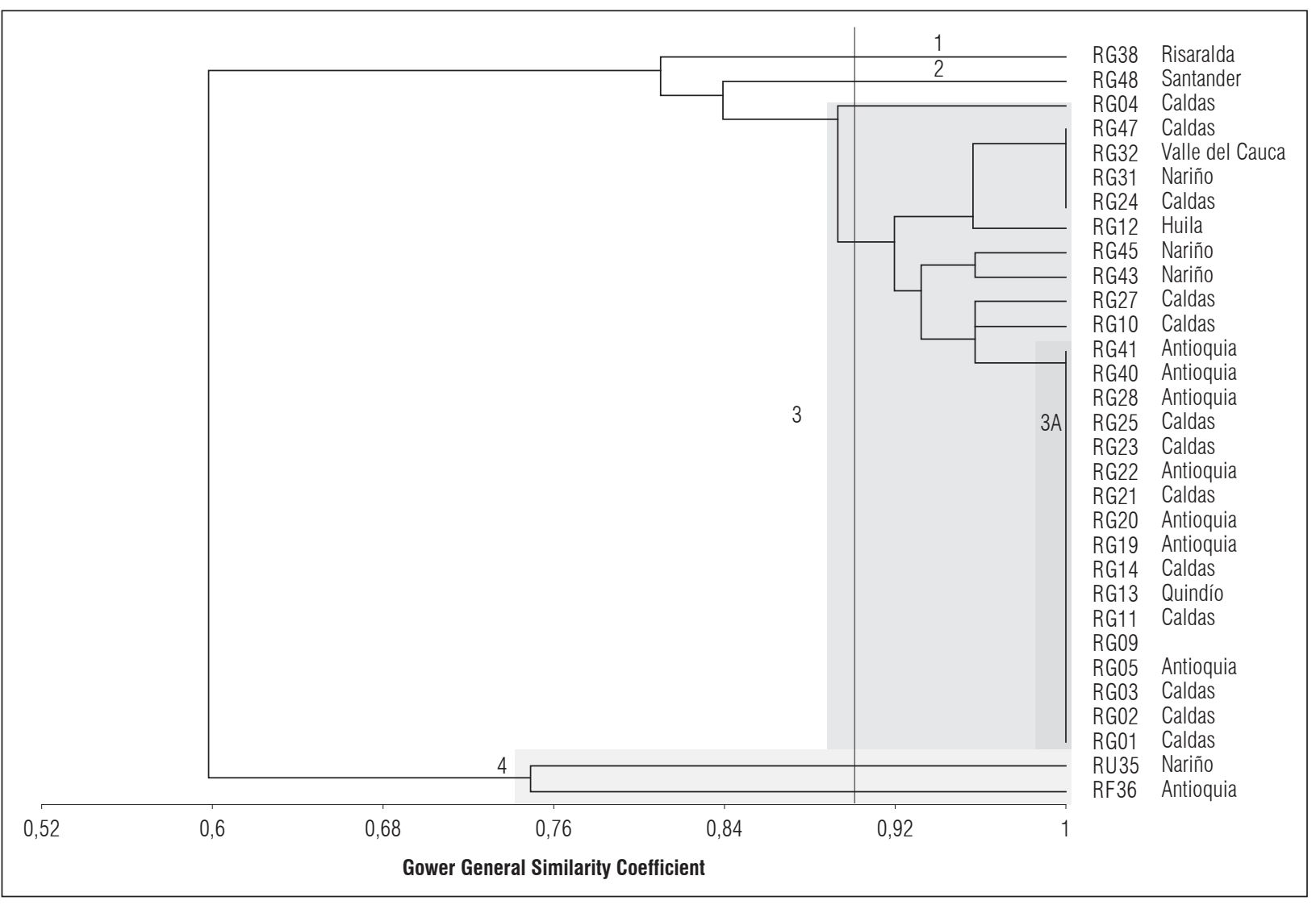

Figura 2. Dendrograma obtenido por análisis de coordenadas principales para 31 accesiones de Rubus spp., a partir de 26 variables cualitativas con MVSP 3.13 .

\begin{tabular}{|c|c|c|c|c|c|}
\hline Descriptores cualitativos & $\begin{array}{c}\text { Grupo } 1 \\
\text { R. glaucus } \\
\text { Sin espinas }\end{array}$ & $\begin{array}{c}\text { Grupo } 2 \\
\text { R. glaucus } \\
\text { Pie de Cuesta }\end{array}$ & $\begin{array}{l}\text { Grupo } 3 \\
\text { R. glaucus }\end{array}$ & $\begin{array}{l}\text { Grupo 3A } \\
\text { R. glaucus }\end{array}$ & $\begin{array}{c}\text { Grupo } 4 \\
\text { Silvestres }\end{array}$ \\
\hline Cerosidad del tallo* & Presente & Presente & Presente & Presente & Ausente \\
\hline Forma del tallo* & Redondo & Redondo & Redondo & Redondo & Acanalado \\
\hline Pubescencia en el tallo* & Ausente & Ausente & Ausente & Ausente & Presente \\
\hline Forma de aguijones del tallo* & Rudimento & Recurvados & Rectos y Recurvados & Rectos y Recurvados & Rectos y Recurvados \\
\hline Base del aguijón en el tallo* & Angosta & Amplia & Amplia & Amplia & Amplia \\
\hline Forma de los aguijones de la hoja* & Rudimento & Recurvados & Recurvados & Recurvados & Rectos y Recurvados \\
\hline Base del aguijón en la hoja* & Angosta & Amplia & Amplia & Amplia & Amplia \\
\hline Forma de la hoja* & Trifoliada & \begin{tabular}{|l|} 
Trifoliada \\
\end{tabular} & \begin{tabular}{|l|} 
Trifoliada \\
\end{tabular} & Trifoliada & Trifoliada y palmeada \\
\hline Margen de la hoja* & Biserrado & \begin{tabular}{|l|} 
Biserrado \\
\end{tabular} & Biserrado & Biserrado & Serrado \\
\hline Base de la hoja (V) & Subcordada & $\begin{array}{l}\text { Subcordada y } \\
\text { Subtruncada } \\
\end{array}$ & Subtruncada & Subcordada & Obtusa \\
\hline Base de la hoja (R) & Subcordada & Subtruncada & Subcordada & Subcordada & Obtusa \\
\hline Ápice de la hoja* & Acuminado & Acuminado & Acuminado & Acuminado & Agudo \\
\hline Forma del foliolo* & Ovado-elíptico & Ovado-elíptico & Ovado-elíptico & Ovado-elíptico & Ovado-elíptico \\
\hline Color del envés* & Blanco & Blanco & Blanco & Blanco & Verde \\
\hline
\end{tabular}

*En tallo vegetativo y reproductivo; V: tallo vegetativo; R: tallo reproductivo. 
Las accesiones $R$. glaucus presentan una similitud de 0,81 y sus características cualitativas comunes son: la cerosidad del tallo, el tallo redondo, la ausencia de pubescencia en el tallo, el margen de la hoja biserrado, el ápice de la hoja acuminado y el color blanco en el envés de la hoja (Fig. 2; Tab. 4).

El grupo 3 se encuentra constituido por 27 accesiones de $R$. glaucus, provenientes de diferentes departamentos productores de mora. Dentro de este grupo se encuentra una subdivisión denominada 3A, compuesta por 17 materiales en su mayoría provenientes de Antioquia y Caldas, idénticos (similitud igual a 1) en todas las características cualitativas entre ellos. La característica por la que difieren del resto del grupo 3 es la base de la hoja subcordada en ramas vegetativas, mientras que los demás materiales del grupo la tienen subtruncada (Fig. 2; Tab. 4).

Al grupo 2 pertenece la accesión RG48 "Pie de Cuesta", la cual se diferencia del grupo 3 por presentar solo aguijones recurvados, la base de la hoja en tallo reproductivo es subtruncada en lugar de subcordada, mientras que en el tallo vegetativo la base de la hoja puede ser tanto subcordada como subtruncada. El grupo 1 está compuesto por la accesión RG38 "Sin Espinas", la cual difiere del resto de accesiones $R$. glaucus en la presencia de rudimentos de aguijones, en lugar de los aguijones característicos en especies Rubus, también se diferencia porque la base de estos rudimentos es angosta y no amplia como en las demás accesiones de R. glaucus (Fig. 2; Tab. 4).

\section{DISCUSIÓN}

\section{Análisis de descriptores cuantitativos}

Se encontró que las variables con correlaciones más altas con los cuatro primeros componentes principales, los cuales agrupan el $60 \%$ de la variación total fueron: longitud del peciolo tanto de rama reproductiva como vegetativa, longitud del peciolulo de rama vegetativa, longitud del foliolo de rama reproductiva, número de aguijones por hoja de rama vegetativa y reproductiva, longitud de entrenudos de rama reproductiva, número de frutos de forma primaria o cónicos y número de piezas de la corola. Por tanto, estos descriptores son los que más aportan en la discriminación de las accesiones de la colección en estudio de los materiales de Rubus spp. de la colección en estudio.
Zamorano et al. (2007) reportaron en materiales colectados en Valle, Cauca y Nariño de las especies $R$. glaucus, $R$. urticifolius y $R$. robustus, siete componentes principales agrupando el $78 \%$ de la variabilidad de 22 variables cuantitativas. Las variables en las que coincidieron el análisis de componentes hecho por Zamorano et al. (2007) y el de este estudio debido a que más aportan a la variabilidad en las evaluaciones de materiales Rubus fueron cinco: longitud del peciolo en ramas vegetativas y reproductivas, longitud del peciolulo de ramas vegetativas y longitud de entrenudos de ramas reproductivas. Sin embargo, el estudio de Zamorano et al. (2007) fue relevante el ancho de foliolo de rama vegetativa, mientras que en la colección de Corpoica fue más importante la longitud del foliolo de rama vegetativa y además se incluyeron las variables número de aguijones por hoja en rama vegetativa y reproductiva, resultado similar al reportado por Trivedi et al. (2016) en un estudio de diversidad genética de nueve especies de Rubus en la región del Himalaya Central, quienes encontraron que las características de ancho y largo de los foliolos terminales son las de mayor importancia para la discriminación de las accesiones. A nivel reproductivo no coincidieron las observaciones en ninguna variable, ya que este análisis incluyó los descriptores: número de frutos de forma cónica y número de piezas de la corola, mientras que Zamorano et al. (2007) identificaron peso del fruto, longitud del receptáculo y número de drupas como las variables con mayor aporte en la discriminación de especies de Rubus a nivel reproductivo. De acuerdo con el análisis de componentes principales de Marulanda y López (2009), las variables que agrupan mayor variación en materiales de $R$. glaucus de la zona cafetera colombiana son: longitud del foliolo de rama vegetativa, la cual coincide con los resultados de este estudio en lo relacionado con diámetro del tallo de rama vegetativa y número de ramas vegetativas que no fueron distintivas de la colección examinada.

En la caracterización morfológica realizada por Zamorano et al. (2007), se descartaron las variables número de piezas de la corola y número de piezas del cáliz debido a que no fueron discriminantes en la colección estudiada, pero en esta investigación se incluyeron, siendo la primera discriminante entre las accesiones de la especie cultivada y las accesiones silvestres.

En el dendrograma obtenido a partir del análisis de componentes principales se observó la conformación de tres grupos, el primero se refiere a las dos especies 
silvestres, mientras que los materiales de $R$. glaucus se agruparon en los grupos dos y tres. Sin embargo, el grupo 2 con individuos de $R$. glaucus es más cercano al grupo uno de materiales silvestres que al grupo tres conformado por otros $R$. glaucus. La mayor afinidad entre estos dos grupos se debe principalmente a la similitud en características de las hojas como una menor longitud en peciolo en rama reproductiva, peciolulo en rama vegetativa y foliolo en rama reproductiva comparado con el grupo 3 (Tab. 3). Mientras que los descriptores que tienen en común los grupos dos y tres son: mayor longitud de peciolo en rama vegetativa, menor número de aguijones en ambos tipos de rama, mayor número de frutos de forma cónica y entrenudos en rama reproductiva más cortos que las especies silvestres. Por tanto, pareciera que dentro de $R$. glaucus hay algunas accesiones intermedias con mayor cercanía o introgresiones de las accesiones silvestres y otras que han sido objeto de selección hacia características de interés comercial. Queda claro que hay características que distinguen los materiales silvestres de los cultivados como la disminución en el número de aguijones en hoja, en la longitud de los entrenudos e incremento en el tamaño de órganos fuente como foliolos, peciolos y peciolulos. Zamorano et al. (2007) indican que en su colección los descriptores cuantitativos fueron más útiles para identificar caracteres comunes entre materiales que para distinguir entre especies.

El análisis de componentes principales basado en características cuantitativas evidencia variabilidad a nivel fenotípico entre y dentro de los materiales de Rubus evaluados. Se observa en el agrupamiento a partir del análisis de componentes principales, que hay una tendencia con algunas excepciones, a encontrar accesiones de $R$. glaucus provenientes de Caldas en el grupo 3 con 8 materiales de 15 que lo componen. Por su parte, el grupo 2 involucra en mayor proporción materiales de $R$. glaucus originarios de Antioquia (6 accesiones de 14). Podría considerarse que para la colección en estudio el lugar de origen puede tener alguna influencia por procesos de adaptación a alguna condición ambiental específica o por los criterios de selección a los que haya habido lugar, de manera que se vea reflejado en la respuesta fenotípica de los materiales; las accesiones de Antioquia fueron colectadas en clima frío con altitud promedia superior a 2.500 msnm, mientras que las de Caldas se colectaron en clima frío moderado a $2.100 \mathrm{msnm}$, esta condición diferencial de altitud puede causar, a su vez, un gradiante altitudinal en la vegetación, así en el caso de Rubus en la región del Himalaya Central según Trivedi et al.
(2016) las plantas muestran adaptación específica a ciertas condiciones ambientales, en $R$. ellipticus que crece a $1.200 \mathrm{msnm}$ el índice de área foliar (IAF) es estadísticamente superior a otras especies colectadas a altitudes superiores a $1.480 \mathrm{msnm}$. Según Ellstrand y Rose (1987) a nivel ecológico las diferencias entre genotipos pueden validar el argumento de que diferentes modos de selección pueden estar involucrados en el mantenimiento de la diversidad clonal a nivel local, independiente del modo de reproducción.

\section{Análisis de descriptores cualitativos}

Zamorano et al. (2007) descartaron la característica de color del envés verde por no ser discriminante para su colección con el análisis de componentes principales, pero en esta evaluación al utilizar el análisis de coordenadas principales, esta característica diferenció las especies silvestres $R$. urticifolius y $R$. floribundus de las accesiones de $R$. glaucus que presentaron envés blanco (Tab. 4). Mediante el análisis de agrupamiento basado en el índice de similitud de Dice, Zamorano et al. (2007) obtuvieron tres agrupaciones claras por cada una de las especies en estudio. De igual manera, las tres especies aquí evaluadas ( $R$. glaucus, $R$. urticifolius y $R$. floribundus) se agruparon de manera separada, mostrando mayor cercanía entre sí los individuos de las accesiones $R$. urticifolius y $R$. floribundus.

El análisis de las características cualitativas sugiere que las entradas de $R$. glaucus de la colección tienen una alta similitud y se determinan tres agrupaciones dentro de la población $R$. glaucus. La agrupación 3A en la figura 2 muestra que la característica hoja subcordada es prevalente en materiales provenientes de Caldas y Antioquia y diferencial con respecto a los grupos 1 y 2. Los grupos 1 y 2 se conforman cada uno de una sola accesión que se diferencian de los demás materiales principalmente por características referentes a los aguijones.

Se debe destacar la accesión RG38, la cual parece ser más domesticada ya que se distingue de las demás accesiones por la presencia de rudimentos de aguijón, es decir que el aguijón que se encuentra en la mayoría de materiales de $R$. glaucus ha sufrido una modificación hacia una estructura menos rígida, de manera que las labores de campo y cosecha se facilitan con este tipo de plantas; mientras el resto de características fenotípicas a nivel cualitativo son comunes con las otras entradas de $R$. glaucus. En cuanto a sus características cuantitativas, RG38 no muestra alguna distinción 
notable con los materiales $R$. glaucus, se agrupa en el grupo 3 de la figura 1, que se destaca por tener órganos vegetativos de mayor tamaño (longitud de foliolos, peciolos y peciolulos) lo cual podría verse reflejado en frutos más grandes. En ese mismo sentido, Marulanda y López (2009) indican que los genotipos sin aguijones tienen la misma productividad y tamaño de fruto que los materiales con aguijones. La característica "sin espinas" se ha observado en otras especies de zarzamoras Rubus y a nivel comercial ha tenido importancia en el desarrollo de variedades (Coyner et al., 2005; Clark et al., 2007). En Colombia la característica "Sin Espinas" puede tener un impacto importante en la producción de mora ya que al mejorar la eficiencia en las labores de campo se pueden reducir costos de producción. No obstante, la ausencia de aguijones es una característica que ha mostrado tanto herencia dominante como recesiva (Coyner et al., 2005), por tanto es prioritario conocer el tipo de herencia en los materiales de $R$. glaucus para el uso de estos cultivares como parentales en un programa de mejoramiento.

Según Yang et al. (2010), dentro de las ventajas evolutivas que confiere la poliploidía está el aumento de la heterocigosidad y un mayor conjunto de genes y alelos sobre los cuales puede actuar la selección. En alopoliploides como se considera a $R$. glaucus pueden fijarse las interacciones positivas de genes homólogos entre los genomas alternos, lo cual tiene un comportamiento similar a la heterosis en organismos diploides. La interacción de individuos con este tipo de conformación genética con distintos ambientes puede ser causante de fenotipos alternativos de un genotipo dado con adaptación o no a diferentes condiciones, como lo advierten Ellstrand y Rose (1987) en poblaciones clonales.

Con base en el análisis cualitativo se puede sugerir que la población de $R$. glaucus de la colección puede estar conformada por pocos genotipos con diferentes grados de dispersión, como se ha observado en varias especies de Rubus cuya reproducción es principalmente de carácter apomíctico o vegetativo (Nybom y Schaal, 1990; Korpelainen et al., 1999). De acuerdo con Korpelainen et al. (1999), en poblaciones de $R$. chamaemorus el contraste entre una baja diversidad genética frente a una amplia variabilidad morfológica es de importancia tanto para la domesticación de la especie como para el trabajo en mejoramiento genético, ya que la labor de selección de genotipos sobresalientes tiene una importante influencia de la interacción con el ambiente.

\section{CONCLUSIONES}

Los descriptores morfológicos permitieron hacer distinciones claras entre las tres especies de Rubus en estudio. El análisis de los datos cuantitativos y cualitativos mostraron variabilidad en toda la colección de Rubus a nivel morfológico incluso en los materiales $R$. glaucus. También se identificaron algunas asociaciones entre la respuesta fenotípica y el departamento de origen, de manera que los genotipos $R$. glaucus en particular pueden mostrar variaciones en su respuesta fenotípica según el ambiente en el que han sido objeto de selección. La variabilidad morfológica de la colección de Rubus estudiada es importante para ser utilizada en programas de fitomejoramiento para obtener genotipos de mora con ciertos caracteres vegetativos y reproductivos que permitan incentivar su cultivo en las condiciones ambientales de las principales zonas de producción en Colombia.

\section{Agradecimientos}

Al investigador Juan Carlos Granados Tochoy, Curador del Herbario de la Facultad de Ciencias Agrarias de la Universidad Nacional de Colombia, sede Bogotá, y al profesor Edgar Leonardo Linares Castillo, experto en el género Rubus, del Instituto de Ciencias Naturales de la Universidad Nacional de Colombia, sede Bogotá, por su aporte en la revisión taxonómica de la colección de especies Rubus.

\section{REFERENCIAS BIBLIOGRÁFICAS}

Alice, L.A. y C.S. Campbell. 1999. Phylogeny of Rubus (Rosaceae) based on nuclear ribosomal DNA internal transcribed spacer region sequences. Am. J. Bot. 86, 81-97. Doi: $10.2307 / 2656957$

Clark, J.R., E.T. Stafne, H.K. Hall y C.E. Finn. 2007. Blackberry breeding and genetics. Plant Breed. Rev. 29, 17146. Doi: 10.1002/9780470168035.ch2

Coyner, M.A., R. Skirvin, M.A. Norton y A.G. Otterbacher. 2005. Thornlessness in blackberries: A review. Small Fruits Rev. 4, 83-106. Doi: 10.1300/J301v04n02_09

Decisia. 2003. SPAD Data mining and text mining. Paris.

Ellstrand, N.C. y M.L. Roose. 1987. Patterns of genotypic diversity in clonal plant species. Am. J. Bot. 74, 123131. Doi: $10.2307 / 2444338$

Finn, C. 2008. Rubus spp., blackberry. pp. 348-351. En: Janick, J. y R.E. Paull (eds.). The encylopedia of fruits and nuts. CABI, Cambridge, MA. 
Franco, T.L. y R. Hidalgo. 2003. Análisis estadístico de datos de caracterización morfológica de recursos fitogenéticos. Bol. Técn. 8. Instituto Internacional de Recursos Fitogenéticos (IPGRI), Cali, Colombia.

Gower, J.C. 1971. A general coefficient of similarity and some of its properties. Biometrics 27, 857-871. Doi: $10.2307 / 2528823$

Korpelainen, H., K. Antonius-Klemolay G. Werlemark. 1999. Clonal structure of Rubus chamaemorus populations: comparison of different molecular methods. Plant Ecol. 143, 123-128. Doi: 10.1023/A:1009898209220

Kovach, W.L. 1999. MVSP: Multivariate Statistical Package for Windows, 3.13 ed. Kovach Computing Services, Wales.

Marulanda, M. y A. López. 2009. Characterization of thornless Rubus glaucus in Colombia. Can. J. Pure Appl. Sci. 3, 875-885.

Marulanda, M., A.M. López y S. Aguilar. 2007. Genetic diversity of wild and cultivated Rubus species in Colombia using AFLP and SSR markers. Crop Breed. Appl. Biotechnol. 7, 242-252. Doi: 10.12702/1984-7033. v07n03a03

Marulanda, M. y M.d.P. Márquez. 2001. Caracterización de la diversidad de Rubus glaucus Benth. con marcadores moleculares RAPD. Actual. Biol. 23, 57-63.

MADR (Ministerio de Agricultura y Desarrollo Rural. 2012. Anuario estadístico de frutas y hortalizas 2007-2011 y sus calendarios de siembras y cosechas. Resultados Evaluación Agropecuaria Municipal 2011. Ed. J.L. Impresores Ltda., Bogotá.

Morillo, A.C., Y. Morillo, J.E. Muñoz, H.D. Vásquez y A. Zamorano. 2005. Caracterización molecular con microsatélites aleatorios RAMs de la colección de mora, Rubus spp., de la Universidad Nacional de Colombia sede Palmira. Acta Agron. 54, 1-15.
Nybom, H., S.H. Rogstad y B.A. Schaal. 1990. Genetic variation detected by use of the M13 "DNA fingerprint" probe in Malus, Prunus, and Rubus (Rosaceae). TAG Theor. Appl. Genet. 79, 153-156. Doi: 10.1007/ BF00225944

Patel, A.V., J. Rojas-Vera y C.G. Dacke. 2004. Therapeutic constituents and actions of Rubus species. Curr. Med. Chem.11,1501-1512.Doi: 10.2174/0929867043365143

Patiño, V.M. 2000. Historia y dispersión de los frutales nativos del neotrópico. Vol. 326. Cali, Colombia.

Romoleroux, K. 1996. Rosaceae. pp. 169. En: Harling, G. y L. Annderson (eds.). Flora del Ecuador. Vol. 56. University of Goteborg, Gothenburg, Suecia.

Stafne, E.T. y J.R. Clark. 2004. Genetic relatedness among eastern North American blackberry cultivars based on pedigree analysis. Euphytica 139, 95-104. Doi: 10.1007/s10681-004-2436-4

Trivedi, A.K., S.K. Verma y R.K. Tyagi. 2016. Variability in morho-physiological traits and antioxidant potential of Rubus species in Central Himalayan Region. Ind. Crops Prod. 82, 1-8. Doi: 10.1016/j. indcrop.2015.12.022

Ward, J. 1963. Hierarchical grouping to optimize an objective function. J. Amer. Stat. Assoc. 58, 236-244. Doi: 10.1080/01621459.1963.10500845

Yang, X., C.-Y. Ye, Z.-M. Cheng, T. Tschaplinski, S. Wullschleger, W. Yin, X. Xia y G. Tuskan. 2010. Genomic aspects of research involving polyploid plants. Plant Cell Tissue Organ Cult. 1-11. Doi: 10.1007/ s11240-010-9826-1

Zamorano, A., A.C. Morillo, Y. Morillo, H. Vásquez y J. Muñoz. 2007. Caracterización morfológica de mora en los departamentos de Valle del Cauca, Cauca y Nariño, de Colombia. Acta Agron. 56, 51-60. 